\title{
Comment on "Is Uric Acid elevation a random finding or a causative agent of diabetic nephropathy?"
}

Dear Editor,

We read with great interest the study by Kocak et al. ${ }^{1}$ in which they demonstrated that UA levels may be an important predictor of nephropathy in diabetic patients. Although this article is interesting, in our opinion, there are also some concerns that should be addressed.

To begin with, the authors should give the definition of diabetic nephropathy. Additionally, the diagnosis criteria for diabetic nephropathy should be referenced. In table 1, we believe the mean with the corresponding statistics deviation may be more adequate for systolic blood pressure, diastolic blood pressure, body mass index, and so on. In general, the above variables should have a normal distribution. If the variables fail to show normal distribution, more samples should be included in the study.

Secondly, when you study the relationship between uric acid and diabetic nephropathy, the subjects included should be subjects with diabetic nephropathy and normal controls. The changes in uric acid may change with the process of diabetic nephropathy development.

\section{REFERENCE}

1. Kocak MZ, Aktas G, Duman TT, Atak BM, Savli H. Is uric acid elevation a random finding or a causative agent of diabetic nephropathy? Rev Assoc Med Bras (1992). 2019;65(9):1155-60. 